\title{
Prevalence of rifampicin resistant Mycobacterium tuberculosis among presumptive tuberculosis patients in selected governmental hospitals in Addis Ababa, Ethiopia
}

\author{
Balew Arega ${ }^{1 *}\left(\mathbb{D}\right.$, Fiqrte Menbere ${ }^{3}$ and Yitagesu Getachew ${ }^{2}$
}

\begin{abstract}
Background: Rapid detection of rifampicin resistance is essential for early management and prevention of transmission of multidrug-resistant tuberculosis (MDR-TB). We studied the prevalence of rifampicin resistance of Mycobacterium tuberculosis (MTB) among presumptive TB patients in Addis Ababa, Ethiopia.

Methods: A retrospective cross sectional study was conducted in three referral hospitals and the regional laboratory in Addis Ababa city from March 2015 to October 2017. Data was collected by data-extraction sheet from registration books. It was analyzed using SPSS version 20. Statistically significant association was taken with $P<0.05$.

Results: A total of 12,414 (11,672 adults and 742 paediatrics) TB presumptive patients were included in the study. The overall prevalence of TB was 15.11\% (1876/12414) in all age groups and 13.6\%(101/742) among paediatric population. Rifampicin resistant TB was 9.9\% (186/1876) in all TB confirmed cases and 7.9\% (8/101) in paediatric TB patients. The prevalence of rifampicin resistant TB among new and previously treated was 7.6 and 27.4\%, respectively. Sex (being female) and previous TB treatment were significantly associated with rifampicin resistant TB.

Conclusions: Rifampicin-resistant TB is prevalent both among adult and paediatric TB patients. The strong association of rifampicin resistance with previous treatment in this study suggests the need to improve and monitor the treatment to limit the emergence of drug resistant TB.
\end{abstract}

Keywords: MTB prevalence, Rifampicin resistance, MDR-TB, Addis Ababa, Ethiopia

\section{Background}

The emergence and spread of multidrug-resistant tuberculosis (MDR-TB) is threat, which complicate diagnosis, treatment and control of the disease [1]. On average, 4.1\% of newly diagnosed and 19\% previously treated TB patients are estimated to MDR-TB worldwide in 2017 [2].

Empirical TB treatment without drug susceptibility testing (DST), which is a common practice in many developing countries, is believed to increase the day to day risk of transmission of drug resistant strains [3, 4]. Therefore, routine testing of all patients with $\mathrm{TB}$ is

\footnotetext{
* Correspondence: balewmlt@gmail.com

${ }^{1}$ Yekatit 12 Hospital Medical College, P.O.Box 257, Addis Ababa, Ethiopia Full list of author information is available at the end of the article
}

widely recognized as the most appropriate surveillance approach for monitoring trends in drug resistant TB [5].

WHO endorsed the Xpert MTB/RIF assay, which is a rapid and automated molecular system that detects both M.tuberculosis DNA and rifampicin-resistance (RR) associated mutations simultaneously. Research recognized that RR can be a surrogate marker for MDR-TB in more than $90 \%$ of the cases [6]. Hence, WHO recommends that RR-TB patients should be treated like patients with MDR-TB [7]. Initially, this technique was indicated for patients with TB/HIV co-infection, presumptive MDRTB and paediatrics TB patients [8] but 3 years later of its implementation, it was recommended for all patients suspected of having TB [9]. Ethiopia ranks 10th among 
the high-TB-pandemic countries and 15th among the 27 high MDR-TB countries with more than 5000 estimated MDR-TB patients each year [10]. A recent national drug resistance survey conducted in Ethiopia reported a 2 and $11 \%$ prevalence of MDR-TB among new and pretreatment cases, respectively [11]. In addition, a meta-analysis and systematic review conducted in our country reported that drug resistant $\mathrm{TB}$ rates were stable in the last 10 years [12]. Drug resistant TB treatment is more complex than susceptible TB due to longer treatment time, increased toxicity and costs [13].

In Ethiopia, sputum smear microscopy is the commonly used laboratory diagnostics technique for TB. Mycobacterium tuberculosis culture, the gold standard test, is limited only to regional laboratories and primarily used for research purposes. The Xpert MTB/RIF assay is now going to be implemented in all health facilities, mainly in referral hospitals across the country since 2014, as per the recommendation of WHO [14]. This survey assessed the prevalence of RR-TB among presumptive TB patients diagnosed using Xpert MTB/RIF assay in selected governmental hospitals in the capital city of Ethiopia.

\section{Methods}

\section{Study design, area and period}

This was a health facility based retrospective cross-sectional study conducted from March 2015 to October 2017 in three referral governmental hospitals and the regional laboratory found in Addis Ababa Administrative health office. The study population were all TB presumptive (patients with clinical signs and symptoms suggestive of $\mathrm{TB}$ ) patients who visited the study area during the study period.

\section{Laboratory investigation and data collection}

A single sputum sample per patient for age greater than 6 years and a gastric aspirate sample in case of children less than this age group were used in all study facilities for the diagnosis of all presumptive TB patients using Xpert MTB/RIF assay. Samples were collected before the patients started anti-TB treatment. Samples were processed by GeneXpert MTB/ RIF assay. These were diluted and decontaminated, and the GeneXpert MTB/RIF assay was performed according to the manufacturer's manual. Other laboratory diagnostic methods such as culture and acid fast bacilli (AFB) smear microscopy were not done for patients with M.tuberclosis negative result by Xpert MTB/RIF. Patients' records that had incomplete data, e.g., age, gender, Xpert MTB/RIF results, HIV status, sample type, and TB treatment history were excluded from the study.

\section{Data processing, analysis}

The data was entered in to EPI info version 7 and then exported to SPSS version 20 for analysis. Descriptive statistics was done for the calculation of frequencies of each variable. All the variables (Age, sex, previous TB treatment history, HIV) included in this study are traditional/known risk factors of drug resistance TB and included in the regression model irrespective of their value.

\section{Ethical issue}

The study was approved by Yekatit 12 Hospital Medical College and Addis Ababa Administrative Health office ethical review committee (ERC). A letter of cooperation was written to each study areas authority and official permission was obtained. Informed consent was not sought from the study participants as it used secondary data. Confidentiality of the information collected was maintained by omitting their name and other personal identifiers from extraction sheet.

\section{Result \\ Sociodemographic and clinical characteristics of the studied sample}

Of the 15,099 presumptive TB patients that have submitted samples for TB diagnosis, 12,414 (82.2\%) had complete data and were included in the study. Of these, $11,672(94 \%)$ had age $>15$ years and $742(6 \%)$ had age $\leq$ 15 years (paediatric). The overall prevalence of MTB detected using Xpert MTB/RIF assay was 15.11\% (1876/ $12414)$ in all age groups and $13.6 \%$ (101/742) among paediatric presumptive TB patients. The mean age of the tuberculosis diagnosed patients was $33.33 \pm 13.99$ years, of which 1107 (59\%) were males and 769(41\%) were females. Of 101 pediatric TB patients, 58/101 (57.4\%) were in age range of $11-15$ years. The HIV status was unknown for the majority of the patients (89.7\%) and $11.7 \%(219 / 1876)$ had previously TB treatment history (Table 1).

\section{Detection of rifampicin resistant $M$. tuberculosis}

The overall prevalence of RR-MTB was 9.9\% (186) in all TB diagnosed patients. In paediatric TB patients, 8 (7.9\%) were RR-MTB positive. In this population (Table 2$)$, previously TB treatment $(P<0.001)$ and female sex $(P<0.02)$ were found independently associated with RR-TB. In this population (Table 2 ), previously TB treatment $(P<0.001)$ and female sex $(P<0.02)$ were found independently associated with RR-TB. The prevalence of RR-MTB was almost equal among HIV positive and negative patients $(14.5 \%$ vs $14.3 \%$ respectively). There were no significant differences in the prevalence of RR-MTB between patients under 15 years and above 15 years $(p>0.05)$. 
Table 1 Sociodemographic and clinical characteristics of tuberculosis patients in Addis Ababa, Ethiopia $(N=1876)$

\begin{tabular}{lll}
\hline Variables & Total TB patients & Percentage (\%) \\
\hline Sex & 1107 & 59 \\
Male & 769 & 41 \\
Females & & \\
Age group & 30 & 1.6 \\
$0-5$ & 13 & 0.7 \\
6-10 & 58 & 3.1 \\
$11-15$ & 863 & 46 \\
16-30 & 584 & 31.1 \\
31-45 & 250 & 13.3 \\
$46-60$ & 78 & 4.2 \\
$>61$ & & \\
History TB treatment & 193 & 10.3 \\
Relapse & 17 & 0.91 \\
Failure & 9 & 0.5 \\
Lost to follow up & 1657 & 88.3 \\
New cases & & 8.7 \\
HIV status & 117 & 4.1 \\
Positive & 77 & \\
Negative & 1682 & \\
Unknown & & \\
\hline
\end{tabular}

HIV Human immunodeficiency virus

\section{Discussion}

In the present study, the prevalence of $M$. tuberculosis infection was found to be $15.11 \%$. Other several studies reported quite a diverse magnitude of $M$. tuberculosis (15.4-25\% [15-19] in the different parts of the country. This might be related to variation in risk for HIV acquisition, geographical variation, method of diagnosis, study setting or TB control practice.

The prevalence of M.tuberclosis among pediatrics TB Patients (13.6\%) in the current study is comparable to a report from South Africa (13\%) [20] and Uganda 14\% [21]. Yet, it is lower than a study done in Southwest Ethiopia (31.7\%) [22]. This substantial difference can be owing to the methods of diagnosis. While we used only Xpert MTB/RIF assay, the other study applied microscopy and culture and other molecular techniques for identification.

The existence of RR-TB is a serious health problem in the study population. The prevalence of RR-TB in this study is in line with previous studies in Ethiopia (910.3\%) $[17,19]$ and Nigeria (12.1\%) [23]. However, other studies in the country reported a lower (5.7-7.5\%) $[24,25]$ and a higher $(15.8-33.3 \%)[18,26,27]$ level of RR-TB. The variation of RR-TB across the country might be related to differences in patient selection, sample size (small sampling could generate higher resistance rate).

In the present study, the proportion of RR-MTB (7.9\%) among paediatrics TB patients was consistent to two studies in China $(6.9-8.3)[28,29]$ yet higher than a

Table 2 Prevalence of rifampicin resistant TB in TB patients based on sex, gender, age, treatment history, and HIV status in Addis Ababa, Ethiopia $(N=1876)$

\begin{tabular}{|c|c|c|c|c|c|}
\hline Variables & RR-TB N (\%) & Not RR-TB N (\%) & COR $(95 \% \mathrm{Cl})$ & $\mathrm{AOR}(95 \% \mathrm{Cl})$ & $P$ Value \\
\hline \multicolumn{6}{|l|}{ Gender } \\
\hline Male & $95(14.6) \%$ & $1012(85.8)$ & reference & & \\
\hline Females & $91(11.8)$ & $678(88.2)$ & $1.43(1.06-1.52)$ & $1.5(1.10-1.99)$ & 0.02 \\
\hline \multicolumn{6}{|l|}{ Age-group years } \\
\hline $0-15$ & $8(7.9)$ & $93(92.1)$ & reference & & \\
\hline $16-30$ & $94(10.9)$ & $769(89.1)$ & $1.421(0.67-3.02)$ & & \\
\hline $31-45$ & $60(10.3)$ & $524(89.7 \%)$. & $1.33(0.62-2.88)$ & & \\
\hline $46-60$ & $17(6.8)$ & $233(93.2)$ & $0.85(0.35-2.03)$ & & \\
\hline$>61$ & $7(9)$ & $71(91)$ & $1.15(0.39-3.31)$ & & \\
\hline \multicolumn{6}{|l|}{ TB-treatment history } \\
\hline Previously Rx cases & $60(27.4)$ & $159(72.6)$ & $4.6(3.24-6.49)$ & $4.7(3.30-6.75)$ & 0.001 \\
\hline New cases & $126(7.6)$ & $1531(92.4)$ & reference & & \\
\hline \multicolumn{6}{|l|}{ HIV status } \\
\hline Positive & $17(14.5)$ & $100(85.5)$ & $1.64(0.956-2.81)$ & & \\
\hline Negative & $11(14.3)$ & $66(85.7)$ & $1.61(0.832-3.12)$ & & \\
\hline Unknown & $158(9.4)$ & $1524(90.6)$ & reference & & \\
\hline
\end{tabular}


study in Uganda (5.7\%) [21] and in Thailand (5.1\%) [30]. This study also found no significant differences $(P>$ $0.05)$ for the presence of RR-TB among pediatrics $(<15)$ and adults. In the same way, other studies [31, 32] also support our findings. Since the largest source of drug resistance $\mathrm{TB}$ comes from adults, it seems reasonable to treat the paediatrics in similar regimen as of their contacts [33, 34]. However, children can acquire infection independently in the community [35], in case of TB high burden settings. Therefore, molecular typing of isolates from pediatrics TB patients and their potential contacts is essential to determine the source of transmission.

In the present study, RR-TB was significantly higher among previously treated patients compared to treatment naive patients. This is in agreement with other studies in Ethiopia (18-19). However, the level of RR-TB among previously untreated cases $(7.6 \%)$ in the analysis is higher than that reported in the latest Ethiopia national survey report (2\%) [36], other study in the country (6.7\%) [19], and in the WHO 2017 report (4.1\%) [2]. The high prevalence of RR-TB among new TB cases in the current study suggests the existence of active personto-person transmission or the existence of undiagnosed new RR-TB cases. In addition, drug resistance among previously untreated cases indicate the performance of TB control program in the past. The strict practice of direct observed therapy (DOTS) and DOTS-plus program currently run-through in Ethiopia is questionable.

According to our study, being female was identified as an independent risk factor for RR-TB. This is in agreement with the report from previous study in Ethiopia [27], another study in Georgia [37], Russia [38] and Estonian patients [39]. The prevalence of RR-TB among females might be related to socioeconomic factors, probably due to lack of control of financial resources at household levels), poor knowledge regarding TB as seen in other studies in country [40] and delay and poor health seeking behavior in females [41]. Since mothers are primary care giver of children in our country, it may increase risk of RR-TB among children. This is very valuable and instructive, and could aid in comprehension for health professionals and policy makers to address the problem. In this study we could not do microbiological confirmation of tuberculosis, phenotypic rifampicin resistance and resistance to other anti-TB drugs.

\section{Conclusion}

In our setting, RR-MTB is prevalent both among adult and paediatric TB patients. Being female and previous treatment with anti-TB drugs was found significantly associated with rifampicin resistance. The strong association of rifampicin resistance with previous treatment suggests the need of an improved monitoring of treatment to limit the emergence of drug resistant MTB strains.

\section{Abbreviations}

HIV: Human immunodeficiency virus; MDR-TB: Multidrug resistant tuberculosis; RR-MTB: Rifampicin resistant Mycobacterium tuberculosis; RRTB: Rifampicin resistant tuberculosis; TB: Tuberculosis; WHO: World Health Organization

\section{Acknowledgments}

The authors are grateful to all laboratory staff members of the Ras Desta Memorial hospital, Yekatit 12 Hospital Medical Colleges, Zewuditu Memorial Hospital and Addis Abeba Regional laboratory for their kind collaboration during the data collection. We would also like to thank the medical college of Yekatit 12 Hospital for the financial support.

\section{Funding}

This study was financially supported by the Yekatit 12 Hospital Medical College. The funder had no role in study design, data collection, and analysis, decision to publish, or preparation of the manuscript.

\section{Availability of data and materials}

All the data are already found in the manuscript and there are no supplementary files. The original data supporting this finding will be available at any time upon request of the corresponding author.

\section{Authors' contributions}

$B A$ and $Y G$, were involved in the study conception and design, data analysis and drafting of the manuscript. FM involved in writing up of the manuscript and data collection. All authors have read and approved the final version of the manuscript.

Ethics approval and consent to participate

The study was approved by Yekatit 12 Hospital Medical College and Addis Ababa Administrative Health office ethical review committee (ERC). A letter of cooperation was written to thee ach study areas authority and official permission was obtained. Informed consent was not sought from study participants as it used secondary data. Confidentiality of the information collected was maintained by omitting their name and other personal identifiers from extraction sheet.

\section{Consent for publication}

Not applicable.

\section{Competing interests}

The authors declare that they have no competing interests.

\section{Publisher's Note}

Springer Nature remains neutral with regard to jurisdictional claims in published maps and institutional affiliations.

\section{Author details}

${ }^{1}$ Yekatit 12 Hospital Medical College, P.O.Box 257, Addis Ababa, Ethiopia. ${ }^{2}$ Department of internal Medicine, Yekatit 12 Hospital Medical College, P.O. Box 257, Addis Ababa, Ethiopia. ${ }^{3}$ Department of Public health research emergency management core process, Addis Ababa Administrative health office regional laboratory, P.O.Box 3-30378, Addis Ababa, Ethiopia.

Received: 7 September 2018 Accepted: 29 March 2019

Published online: 04 April 2019

\section{References}

1. WHO/IUATLD. Global Project on Anti-tuberculosis Drug Resistance Surveillance. Anti-Tuberculosis Drug Resistance in the World. Geneva: WHO; 2008.

2. WHO. Global Tuberculosis Report: 2017.

3. Zignol M, Dean AS, Falzon D, et al. Twenty Years of Global Surveillance of Anti tuberculosis-Drug Resistance. N Engl J Med. 2016;375:11.

4. Shenoi S, Friedland G. Extensively drug-resistant tuberculosis: a new face to an old pathogen. AnnuRev Med. 2009;60:307-20.

5. Snider DE, Kelly GD, Cauthen GM, et al. Infection and disease among contacts of tuberculosis cases with drug resistant and drug susceptible bacilli. Am Rev Respir Dis. 1985;132:125-32. 
6. Riordan P, Schwab U, Logan S, et al. Rapid molecular detection of rifampicin resistance facilitates early diagnosis and treatment of multi-drug resistant tuberculosis. PLOS ONE. 2008;3:1-7.

7. WHO: treatment guidelines for drug-resistant tuberculosis, 2016 update. October 2016 revision.

8. WHO. Rapid implementation of the Xpert MTB/RIF diagnostic test. Geneva: WHO; 2011

9. WHO: Xpert MTB/RIF assay for the diagnosis of TB Meeting Report 2016.

10. Ethiopia Federal Ministry of Health. Guideline for program and clinical management of drug-resistant tuberculosis. 5th ed. Addis Ababa: FMOH; 2009.

11. Kebede AH, Alebachew Z, Tsegaye F, et al. The first Ethiopian National Population-based TB prevalence survey. Int J Tuberc Lung Dis. 2014;18(6): 635-9.

12. Eshetie S, Gizachew M, Dagnew M. Multidrug resistant tuberculosis in Ethiopian settings and its association with previous history of antituberculosis treatment: a systematic review and meta-analysis. BMC Infect Dis. 2017;17:219.

13. WHO. Drug-resistant tuberculosis now at record levels. Geneva: WHO press; 2010

14. Implementation Guldellne for Gene Xpert mtB/rlf assay In ethlopla June, 2014 | Addis Ababa.

15. Alemu A, Tesfay E, Yaregal Z, et al. Status of Xpert MTB/RIF Assay Implementation in Ethiopia. J Infect Dis. 2017:5:6.

16. Hordofa MW, Adela TB. Prevalence of Refampcin mono resistant Mycobacterium tuberculosis among suspected cases attending at Yirgalem hospital. Clin Med Res. 2015;4(3):75-8.

17. Assefa D, Cherinet $Y$, Negussie A. Xpert MTB/RIF assay for diagnosis of pulmonary tuberculosis in sputum specimens in remote health care facility. BMC Microbiol. 2015;15:220.

18. Jaleta FK, Gizachew M, Gelaw B, et al. Rifampicin-resistant Mycobacterium tuberculosis among tuberculosis-presumptive cases at University of Gondar Hospital, northwest Ethiopia. Infect Drug Resist. 2017;10:185-92.

19. Mulu W, Abera B, Yimer M, et al. Rifampicin-resistance pattern of Mycobacterium tuberculosis and associated factors among presumptive tuberculosis patients referred to Debre Markos referral hospital, Ethiopia: a cross-sectional study. BMC Res Notes. 2017;10:8.

20. Nicol MP, Workman L, Isaacs W, et al. Accuracy of the Xpert MTB/RIF test for the diagnosis of pulmonary tuberculosis in children admitted to hospital in Cape Town, South Africa: a descriptive study. Lancet Infect Dis. 2011;11(11): 819-24.

21. Sekadde MP, Wobudeya E, Joloba ML, et al. Evaluation of the Xpert MTB/RIF test for the diagnosis of childhood pulmonary tuberculosis in Uganda: a cross-sectional diagnostic study. BMC Infect Dis. 2013;13:133.

22. Workalemahuet B, Berg S, Tsegaye W, et al. Genotype diversity of Mycobacterium isolates from children in Jimma, Ethiopia. BMC Res Notes. 2013;6:352.

23. Audu ES, Gambo MS, Yakubu AA. Rifampicin resistant mycobacterium tuberculosis in Nasarawa State, Nigeria. Niger J Basic Clin Sci. 2017;14:21-5

24. Hamusse SD, Teshome D, Suaudi MH. Primary and secondary antituberculosis drug resistance in Hitossa District of Arsi Zone, Oromia Regional State, Central Ethiopia. BMC Public Health. 2016;16:59.

25. Tessema B, Beer J, Merker M, et al. Molecular epidemiology and transmission dynamics of Mycobacterium tuberculosis in Northwest Ethiopia: new phylogenetic lineages found in Northwest Ethiopia. BMC Infect Dis. 2013;13:1.

26. Mulisaa G, Workneha T, Hordofa N, et al. Multidrug-resistant Mycobacterium tuberculosis and associated risk factors in Oromia Region of Ethiopia. Int J Infect Dis. 2015;39:57-61.

27. Nigus DM, Lingerew WM, Beyene BA, et al. Prevalence of multi drug resistant tuberculosis among presumptive multi drug resistant tuberculosis cases in Amhara National Regional State, Ethiopia. J Mycobac Dis. 2014;4:152.

28. Tao N, He X. Zhang X etal drug-resistant tuberculosis among children, China, 2006-2015. Emerg Infect Dis. 2017;23(11):1805.

29. Lu J, Li H, Dong F, et al. The Feasibility of Xpert MTB/RIF Testing to Detect Rifampicin Resistance among Childhood Tuberculosis for Prevalence Surveys in Northern China. BioMed Research International. 2017:10 https://doi.org/10.1155/2017/5857369

30. Vandepitte WP, Rattanasataporn R, Treeratweeraphong V. Drug-Resistant Tuberculosis Among Urban Thai Children: A 10-Year Review: Drug-Resistant Tuberculosis In Urban Thai Children. A 10-YEAR REVIEW. South East Asian J Trop Med public health. 2015;46(5):893-9.
31. Zignol M, Sismanidis C, Falzon D. WHO, Multidrug-resistant tuberculosis in children: evidence from global surveillance. Eur Respir J. 2013;42:701-7.

32. Nair SA, Raizada N, Sachdeva KS, et al. Factors Associated with Tuberculosis and RifampicinResistant Tuberculosis amongst Symptomatic Patients in India: A Retrospective Analysis. PLoS ONE. 2016;11(2):e0150054. https://doi. org/10.1371/journal.pone.0150054.

33. World Health Organization. Treatment of tuberculosis: guidelines, 4thedn. WHO/HTM/TB/2009420; 2009.

34. Rachow A, Clowes P, Saathoff E, Mtafya B, Michael E, Ntinginya EN, et al. Increased and expedited case detection by Xpert MTB/RIF assay in childhood tuberculosis: a prospective cohort study. Clin Infect Dis. 2012;54:1388-96.

35. Swaminathan S, Banu Rekha B. Pediatric Tuberculosis: Global Overview and Challenges. Clinical Infectious Diseases. 2010;50(S3):S184-94.

36. Divani N, Zangaladze E, Volkova N, et al. High prevalence of multi-drug resistant tuberculosis in Georgia. Int J Infect Dis. 2008;12:635-44.

37. Toungoussova S, Caugant DA, Sandven P, Mariandyshev AO, Bjune G. Drug resistance of Mycobacterium tuberculosis strains isolated from patients with pulmonary tuberculosis in archangels, Russia. Int J Tuberc Lung Dis. 2002; 6(5):406-14.

38. Lockman S, Kruuner A, Binkin N, Levina K, Wang Y, Danilovitsh M, et al. Clinical outcomes of Estonian patients with primary multi- drug-resistant versus drug-susceptible tuberculosis. Clin Infect Dis. 2001;32:373-80.

39. Bati J, Legesse M, Girmay MG. Community's knowledge, attitudes and practices about tuberculosis in Itang special district, Gambella Region, South Western Ethiopia. BMC Public Health. 2013:13:734.

40. Storla DG, Yimer S, Bjune AG. A systematic review of delay in the diagnosis and treatment of tuberculosis. BMC Public Health. 2008:8:15.

41. Wondimu T, W/Michael K, et al. Delay in initiating tuberculosis treatment and factors associated among pulmonary tuberculosis patients in East Wollega, Western Ethiopia. Ethiop J Health Dev. 2007;21(2):148-56.

\section{Ready to submit your research? Choose BMC and benefit from:}

- fast, convenient online submission

- thorough peer review by experienced researchers in your field

- rapid publication on acceptance

- support for research data, including large and complex data types

- gold Open Access which fosters wider collaboration and increased citations

- maximum visibility for your research: over $100 \mathrm{M}$ website views per year

At $\mathrm{BMC}$, research is always in progress.

Learn more biomedcentral.com/submission 